http://dx.doi.org/10.4046/trd.2012.73.3.169

ISSN: 1738-3536(Print)/2005-6184(Online)

Tuberc Respir Dis 2012;73:169-173

Copyright(C)2012. The Korean Academy of Tuberculosis and Respiratory Diseases. All rights reserved

\title{
Clinical Analysis of Spontaneous Pneumomediastinum
}

Ji-Yoon Ryoo, M.D., Ph.D.

Department of Thoracic and Cardiovascular Surgery, Ilsan Paik Hospital, Inje University College of Medicine, Goyang, Korea

Background: Spontaneous pneumomediastinum is a rare and benign condition that generally occurs in young generations without any precipitating factor or underlying disease. The purpose of this study is to review our experience in dealing with this entity and detail a reasonable course of assessment and management.

Methods: From December 1999 to May 2012, 32 spontaneous pneumomediastinum patients were managed in our hospital. We retrospectively reviewed the result of management.

Results: Patients ranged in age from 10 to 38 years, with the mean age of $18.3 \pm 5.6$ years. Twenty-five patients were men and seven were women. Initial WBC count was 10,039 $\pm 2,993 / \mu \mathrm{L}$ and thirty-one patients underwent computed tomography. Twenty-two patients underwent esophagography and results were shown to be normal. Twenty-five patients were admitted in the hospital and the mean duration of admission was 3.4 \pm 2.0 days. All patients were managed conservatively and discharged without any complications.

Conclusion: Spontaneous pneumomediastinum is a benign condition combined with mild inflammatory signs and secondary causes must to excluded to avoid unfavorable outcomes and select proper management modality.

Key Words: Mediastinum; Mediastinal Emphysema

\section{Introduction}

Spontaneous pneumomediastinum (SPM) is a condition in which air is present in the mediastinum without any definite precipitating causes. It was first described in 1939 by Hamman ${ }^{1}$ and is often referred to as Hamman syndrome. SPM is a relatively rare condition, most commonly seen in young adults. The most common symptoms and signs are chest pain, difficulty in breathing, and subcutaneous emphysema ${ }^{2}$.

SPM results from alveolar rupture induced by high intra-alveolar pressures suddenly rising due to elevated intra-thoracic and intra-abdominal pressures during vigo-

Address for correspondence: Ji-Yoon Ryoo, M.D., Ph.D. Department of Thoracic and Cardiovascular Surgery, Ilsan Paik Hospital, Inje University College of Medicine, 170, Juhwa-ro, Ilsanseo-gu, Goyang 411-706, Korea

Phone: 82-31-910-7366, Fax: 82-31-910-7614

E-mail: ctsriy@paik.ac.kr

Received: Jun. 27, 2012

Revised: Jul. 10, 2012

Accepted: Aug. 29, 2012

(a) It is identical to the Creative Commons Attribution Non-Commercial License (http://creativecommons. org/licenses/by-nc/3.0/). rous exercise, coughing, and meals without definite clinical causes. It is characterized by spontaneous recovery without specific treatments ${ }^{3}$. The author of this study intended to report the results acquired from clinical data analysis, discussion of the literature on patients diagnosed with SPM among pneumomediastinum patients admitted to our hospital from December 1999 to May 2012.

\section{Materials and Methods}

Study population comprised 32 inpatients and outpatients diagnosed with SPM among pneumomediastinum patients admitted our hospital for treatment from December 1999 to May 2012. The study conducted retrospective analysis based on medical history including the age of onset, sex ratio, major symptoms, hospitalization period, clinical examination, and others.

Subjects were excluded in cases of pneumomediastinum caused by underlying diseases or detected after injuries, surgical procedures or other types of invasive procedures. Such patients were considered to have sec- 
ondary pneumomediastinum. SPSS version 10.0 (SPSS Inc., Chicago, IL, USA) was used for statistical analysis and all variables were presented as mean $\pm \mathrm{SD}$.

\section{Results}

Sex ratio was 3.6:1 comprising 25 male and 7 female patients. The age ranged from 10 to 38 and the mean age was $18.3 \pm 5.6$ years old (Table 1). All patients were admitted to the hospital through the emergency room. Chest pain was the most common symptom in $51.2 \%$, followed by neck pain, difficulty in breathing, and sore throat in order (Table 2). According to the medical history, symptoms developed during exercise, meals, study, sleep, cleaning home, and other daily routines (Table 3). According to chest X-ray images, 27 cases manifested the findings of pneumomediastinum along

Table 1. Sex and age of patients

\begin{tabular}{lccc}
\hline Age $(\mathrm{yr})$ & Man & Woman & Total $(\%)$ \\
\hline $10 \sim 19$ & 20 & 4 & $24(75)$ \\
$20 \sim 29$ & 5 & 1 & $6(19)$ \\
$\geq 30$ & 0 & 2 & $2(6)$ \\
Total & $25(78.1)$ & $7(21.9)$ & $32(100)$ \\
\hline
\end{tabular}

Table 2. Patient's symptoms at hospital visit

\begin{tabular}{lrr}
\hline \multicolumn{1}{c}{ Symptoms } & No. & $\%$ \\
\hline Chest pain & 22 & 51.2 \\
Neck pain & 10 & 23.3 \\
Dyspnea & 6 & 14.0 \\
Sore throat & 4 & 9.3 \\
Abdominal pain and vomiting & 1 & 2.3 \\
Total & 43 & 100.0 \\
\hline
\end{tabular}

Table 3. Triggering factors from patient's history

\begin{tabular}{lrr}
\hline \multicolumn{1}{c}{ Factor } & No. & \multicolumn{1}{c}{$\%$} \\
\hline Eating & 3 & 9.4 \\
Exercise & 2 & 6.3 \\
Studying & 2 & 6.3 \\
Sleeping & 1 & 3.0 \\
Nothing or unclear & 24 & 75.0 \\
Total & 32 & 100.0 \\
\hline
\end{tabular}

with heart borders and paratracheal spaces. Among those, nine cases were associated with the findings of subcutaneous emphysema. The other five cases with unclear findings of pneumomediastinum were diagnosed with additional examinations including neck $\mathrm{X}$-ray and computed tomography (CT) of chest and neck. CT and esophagography were performed on 31 and 22 patients, respectively, to verify the confirmation of pneumomediastinum, the scope of incidence, and to rule of the presence of injuries in esophagus, trachea and bronchus resulting secondary pneumomediastinum. Normal findings were found in all cases with esophagography (Table 4). The average white blood cell count was $10,039 \pm 2,993 / \mu \mathrm{L}$ in the blood test performed at the time of visit to emergency room. Fourteen patients showed leukocytosis and four of them had a white blood cells (WBC) count of greater than 15,000/ $\mu \mathrm{L}$ (Table 5). The findings were similar to those of other case study reports ${ }^{2,4}$. Therefore, SPM is thought to be a disease manifesting mild inflammatory responses.

Among the subjects, 25 patients were hospitalized and treatment consisted of bed rest and oxygen therapy.

Table 4. Rdiological examinations methods

\begin{tabular}{lrr}
\hline Diagnostic examination & No. & $\%$ \\
\hline Simple chest X-ray & 32 & 100.0 \\
Neck AP and lateral & 20 & 62.5 \\
Computer tomography & 31 & 96.9 \\
Chest CT only & 26 & \\
Chest and neck CT & 4 & \\
Neck CT only & 1 & \\
Esophagography & 22 & 68.7 \\
\hline
\end{tabular}

CT: computed tomography.

Table 5. Inflammation laboratory findings of patient

\begin{tabular}{lrr}
\hline & No. & $\%$ \\
\hline WBC count $\left(\mu L \mathrm{~L}, 10,039 \pm 2,993^{*}\right)$ & 32 & 100.0 \\
WBC $<10,000$ & 18 & 56.3 \\
$10,000 \leq \mathrm{WBC}<15,000$ & 10 & 31.3 \\
WBC $\geq 15,000$ & 4 & 12.5 \\
\hline
\end{tabular}

*Data: mean \pm standard deviation.

WBC: white blood cells. 
Table 6. Combined disease with pneumomediastinum

\begin{tabular}{lrr}
\hline \multicolumn{1}{c}{ Disease } & No. & $\%$ \\
\hline None & 27 & 84.4 \\
Influenza (H1N1) & 2 & 6.3 \\
Ileus & 1 & 3.1 \\
URI & 1 & 3.1 \\
Bronchitis & 1 & 3.1 \\
Total & 32 & 100.0 \\
\hline
\end{tabular}

URI: upper respiratory infection.

Intravenous fluids were given to a patient who had an associated paralytic ileus. Patients with associated diseases were treated at the same time. Additional antibiotic treatment was not given to patients diagnosed only with SPM.

Seven patients were discharged after their symptoms improved after a period of observation in the emergency room. Consequently, they were observed during follow-up period as outpatients. The average hospitalization period of inpatients was 3.4 \pm 2.0 days.

The improvement of inpatients was examined through daily chest X-ray and patients were discharged when symptoms were disappeared or relieved. Subsequently, the conditions of patients were reexamined one to two weeks later at the outpatient department.

At the time of emergency room visit, five cases had associated diseases and two cases had a past medical history of surgery due to spontaneous pneumothorax (Table 6).

\section{Discussion}

First described in 1827 by Laennec ${ }^{5}$, pneumomediastinum is an abnormal condition of presence of the air or gas within the mediastinum ${ }^{3}$ induced by various reasons. Secondary pneumomediastinum is caused by trauma, infection, injuries on the respiratory and digestive tract. SPM is diagnosed when pneumomediastinum is found with no definite cause ${ }^{6-8}$. SPM was first described in 1939 by Hamman ${ }^{1}$. Pneumomediastinum is diagnosed when a strip or band-shaped black shadow appears in the space between the structures in the me- diastinum and the lungs in a chest X-ray ${ }^{9}$. A number of mechanisms for the pathogenesis have been suggested based on anatomical factors ${ }^{10,11}$.

First, one of the mechanisms is the direct air movement to the mediastinum due to injuries in tracheal bronchus and esophagus. Second, pneumomediastinum may also develop due to air movement from the abdominal cavity to the mediastinum through diaphragm due to bowel perforation, ulcer, diverticulitis, and surgery. Third, it may also occurs due to alveolar rupture generated by infection, aspiration, adult respiratory distress syndrome, pulmonary emphysema, or interstitial lung disease caused by positive pressure ventilation. Fourth, alveolar rupture may also occur due to high intra-alveolar pressure suddenly rising due to elevated intra-thoracic and inta-abdominal pressures caused by Valsalva maneuver, vomiting, coughing, drug inhalation, asthma aggravation, exercise, childbirth, epileptic seizure, or diving. Third and fourth causes were categorized as the causal factors of spontaneous or primary pneumomediastinum in the past, but they are currently regarded as the factor of secondary pneumomediastinum arising from mechanical ventilation and lung diseases.

SPM is a clinically uncommon condition which resolves on its own without specific treatment. SPM is frequently occurs in young males according to case reports where $75 \%$ of patients are males with an average age of $20{ }^{\prime} s^{2}$. When healthy adolescent males, in particular, without preceding history of alcohol or trauma manifest the findings of pneumomediastinum or subcutaneous emphysema in chest X-ray with chest pain, difficulty in breathing, swollen neck, or sore throat, SPM needs to be suspected primarily ${ }^{12}$.

Frequency of SPM was quite low as presented by Abolnik et al. ${ }^{7}$ with $0.003 \%$, Takada et al. ${ }^{13}$ with $0.0095 \%$ Kim et al. ${ }^{14}$ with $0.006 \%$. This is attributable to the failure of detection when the SPM was occurred.

Symptoms of pneumomediastinum vary depending on the amount of trapped air and the inflammations in adjacent tissues. Chest pain occurring due to entered air in the mediastinal space by pulling tissues was $88 \%$ and 
$51 \%$ according to Abolnik et al. ${ }^{7}$ and the author of this study, respectively. Rose et al. ${ }^{3}$ and Abolnik et al. ${ }^{7}$ reported that $50 \%$ had difficulty in breathing generated from compressed trachea due to air present in the mediastinal space. This study detected $14 \%$ with such a manifestation. Pneumomediastinum is hard to differentiate from acute epiglottitis due to almost similar initial symptoms. Similar symptoms such as sore throat and swallowing pain are manifested since air entered into soft tissue spreads and compresses the upper structure of the trachea as dissecting it due to leaked air to the interstitial tissues of blood vessels ${ }^{15}$.

Discriminating symptoms are the presence of high fever, an elevated WBC count, the presence of subcutaneous emphysema, a typical auscultatory crunching sound coinciding with the heart contracting sound (Hamman's sign) and others.

Although SPM is easily diagnosed through radiological examination, Caceres et al. ${ }^{16}$ reported that chest X-ray may not detected the diseases in some cases. Hence, chest CT is thought to be the most reliable diagnostic procedure. Among the case reports from our hospital, patients were confirmed through CT scan of the chest or neck diagnosis except for a case. Secondary pneumomediastinum due to the injury in tracheal bronchus and esophagus and diffuse parenchymal lung disease was excluded through CT. Especially, to exclude pneumomediastinum caused by esophageal injuries, thorough examination on clinical symptoms, history of the present illness, and past medical history are crucial. Furthermore, esophagography was performed to rule out the presence of esophageal perforation or rupture.

Although patients were required to be hospitalized for careful observation, a clinical observation period was followed by emergency department 24-hour observation in cases where patients were unavoidably could not be hospitalized at least. Bed rest and 100\% oxygen administration were initiated for inpatients with a restricted diet. Subsequently, the diet was advanced as symptoms improved during observation period. Pain had disappeared or alleviated within one or two days after it began ${ }^{8,13,17}$. Relieved conditions were showed in the chest X-rays. Although a hospitalization period is determined depending on the conditions of individuals, it is thought to be unnecessary for patients to be hospitalized until complete disappearance of symptoms or confirmation of the full recovery ${ }^{8,13}$.

Although Yellin et al. ${ }^{18}$ and Abolnik et al. ${ }^{7}$ reported recurrent cases, the author of this study did not detect any. Unlike recurrent spontaneous pneumothorax, in general, SPM hardly recurs. Therefore, long-term follow-up period is thought to be unnecessary ${ }^{3}$.

SPM needs to be discriminated from other diseases including acute myocardial infarction, angina pectoris, endocarditis, cardiac tamponade, mediastinitis, dissecting aneurysm, pulmonary embolism, and others ${ }^{14}$.

SPM is a benign disease manifesting mild inflammatory responses ${ }^{16}$. According to the clinical analysis of subjects treated in our hospital, definite causes of pneumomediastinum need to be fully clarified. Patients diagnosed with SPM exhibited findings of good recovery with rest or oxygen therapy without specific additional treatment and complications. However, standardized treatment guidelines are anticipated to be established through further analysis based on more case studies to be utilized in cases where short-term hospitalization or ambulatory care is required in the initial stage of the disease.

\section{References}

1. Hamman L. Spontaneous mediastinal emphysema. Bull Johns Hopkins Hosp 1939;64:1-21.

2. Macia I, Moya J, Ramos R, Morera R, Escobar I, Saumench J, et al. Spontaneous pneumomediastinum: 41 cases. Eur J Cardiothorac Surg 2007;31:1110-4.

3. Rose WD, Veach JS, Tehranzdeh J. Spontaneous pneumomediastinum as a cause of neck pain, dysphagia, and chest pain. Arch Intern Med 1984;144:392-3.

4. Moon HJ. Spontaneous pneumomediastinum: clinical experience of 24 patients in two medical center. Korean J Thorac Cardiovasc Surg 2010;43:663-8.

5. Laennec RT. A treatise on the diseases of the chest and on mediate auscultation. 3rd ed. London: Thomas \& George Underwood; 1829. p. 152-78.

6. Jougon JB, Ballester M, Delcambre F, Mac Bride T, 
Dromer CE, Velly JF. Assessment of spontaneous pneumomediastinum: experience with 12 patients. Ann Thorac Surg 2003;75:1711-4.

7. Abolnik I, Lossos IS, Breuer R. Spontaneous pneumomediastinum: a report of 25 cases. Chest 1991;100: 93-5.

8. Gerazounis M, Athanassiadi K, Kalantzi N, Moustardas M. Spontaneous pneumomediastinum: a rare benign entity. J Thorac Cardiovasc Surg 2003;126:774-6.

9. Keeffe EJ, Jones CF. Pneumomediastinum in the newborn: report of a case. Radiology 1951;56:567-70.

10. Panacek EA, Singer AJ, Sherman BW, Prescott A, Rutherford WF. Spontaneous pneumomediastinum: clinical and natural history. Ann Emerg Med 1992;21: 1222-7.

11. Zylak CM, Standen JR, Barnes GR, Zylak CJ. Pneumomediastinum revisited. Radiographics 2000;20:1043-57.

12. Kim MY, Kim SY, Kim YH, Hwang YJ, Seo JW, Han $\mathrm{YH}$, et al. Idiopathic spontaneous pneumomediastinum: radiologic and clinical features. J Korean Radiol Soc 2004; 51:55-60.

13. Takada K, Matsumoto S, Hiramatsu T, Kojima E,
Watanabe H, Sizu M, et al. Management of spontaneous pneumomediastinum based on clinical experience of 25 cases. Respir Med 2008;102:1329-34.

14. Kim DH, Park JH, Chei CS, Hwang SW, Kim HY, Yoo $\mathrm{BH}$. Spontaneous pneumomediastinum: clinical investigation. Korean J Thorac Cardiovasc Surg 2006;39: 220-5.

15. Caraballo V, Barish RA, Floccare DJ. Pneumomediastinum presenting as acute airway obstruction. J Emerg Med 1996;14:159-63.

16. Caceres M, Ali SZ, Braud R, Weiman D, Garrett HE Jr. Spontaneous pneumomediastinum: a comparative study and review of the literature. Ann Thorac Surg 2008;86: 962-6.

17. Koullias GJ, Korkolis DP, Wang XJ, Hammond GL. Current assessment and management of spontaneous pneumomediastinum: experience in 24 adult patients. Eur J Cardiothorac Surg 2004;25:852-5.

18. Yellin A, Lidji M, Lieberman Y. Recurrent spontaneous pneumomediastinum: the first reported case. Chest 1983;83:935. 